\title{
The melanin-concentrating hormone receptor 1 , a novel target of autoantibody responses in vitiligo
}

\author{
E. Helen Kemp, ${ }^{1}$ Elizabeth A. Waterman, ${ }^{1}$ Brian E. Hawes, ${ }^{2}$ Kim O’Neill, ${ }^{2}$ \\ Raju V.S.R.K. Gottumukkala, ${ }^{1}$ David J. Gawkrodger, ${ }^{3}$ Anthony P. Weetman, ${ }^{1}$ \\ and Philip F. Watson ${ }^{1}$ \\ ${ }^{1}$ Division of Clinical Sciences (North), University of Sheffield, Sheffield, United Kingdom \\ ${ }^{2}$ Department of Central Nervous System/Cardiovascular Biology, Schering-Plough Research Institute, \\ Kenilworth, New Jersey, USA \\ ${ }^{3}$ Department of Dermatology, Royal Hallamshire Hospital, University of Sheffield, Sheffield, United Kingdom \\ Address correspondence to: E. Helen Kemp, Division of Clinical Sciences (North), University of Sheffield, \\ Northern General Hospital, Herries Road, Sheffield, S5 7AU, United Kingdom. \\ Phone: 0114-271-4910; Fax: 0114-256-0458; E-mail: e.h.kemp@sheffield.ac.uk.
}

Received for publication November 15, 2001, and accepted in revised form February 18, 2002.

\begin{abstract}
Vitiligo is a common depigmenting disorder resulting from the loss of melanocytes in the skin. The pathogenesis of the disease remains obscure, although autoimmune mechanisms are thought to be involved. Indeed, autoantibodies and autoreactive T lymphocytes that target melanocytes have been reported in some vitiligo patients. The objective of this study was to identify pigment cell antigens that are recognized by autoantibodies in vitiligo. Using IgG from vitiligo patients to screen a melanocyte cDNA phage-display library, we identified the melanin-concentrating hormone receptor 1 (MCHR1) as a novel autoantigen related to this disorder. Immunoreactivity against the receptor was demonstrated in vitiligo patient sera by using radiobinding assays. Among sera from healthy controls and from patients with autoimmune disease, none exhibited immunoreactivity to MCHR1, indicating a high disease specificity for Ab's against the receptor. Inhibition of $\mathrm{MCH}$ binding to its receptor by IgG from vitiligo patients was also shown.
\end{abstract}

J. Clin. Invest. 109:923-930 (2002). DOI:10.1172/JCI200214643.

\section{Introduction}

Vitiligo is an acquired idiopathic hypomelanotic disorder characterized by circumscribed depigmented macules resulting from the loss of cutaneous melanocytes. Although the exact cause of the condition remains to be established, an autoimmune etiology has been suggested because the disease is frequently associated with other disorders that have an autoimmune origin, such as autoimmune thyroiditis and type 1 diabetes mellitus (1, 2). Furthermore, circulating autoantibodies and autoreactive $T$ lymphocytes that recognize melanocyte antigens are present in the sera of a significant proportion of vitiligo patients compared with healthy individuals (3, 4). Vitiligo autoantibodies are most commonly directed against pigment cell antigens with molecular weights of $35 \mathrm{kDa}, 40-45 \mathrm{kDa}, 75 \mathrm{kDa}, 90 \mathrm{kDa}$, and $150 \mathrm{kDa}$ that are located on the surface of the cell $(5,6)$, but that remain unidentified. In addition, autoantibodies to the melanocyte-specific proteins tyrosinase (7-9), tyrosinaserelated protein-1 (TRP-1) (10), tyrosinase-related protein-2 (TRP-2) (11, 12), and Pmel17 (13), as well as the transcription factor SOX10 (14), have been detected in vitiligo patients. Autoreactive $\mathrm{T}$ cells that recognize MelanA/MART-1, tyrosinase, and Pmel17 have also been reported in individuals with the disease $(4,15-17)$.

The characterization of melanocyte autoantigens in vitiligo is essential for understanding the immunopatho- logical mechanisms in the disease and might enable the development of specific immunological or drug-based therapies for the disorder as well as possible diagnostic assays. Several of the melanocyte autoantigens described in vitiligo are also the targets of immune reactivities in some melanoma patients. The discovery of autoantigens recognized by immune responses in vitiligo may therefore identify antigens that could be useful in the specific immunotherapy of melanoma. For the above reasons, the aim of the present study was to identify novel melanocyte autoantigens in vitiligo using the technique of phage-display based on the pJuFo cloning system $(18,19)$. This technology has been used to identify allergens that bind to human IgE Ab's using yeast and fungal cDNA phagedisplay libraries (20-22). The strategy permits both the expression of cDNA libraries and the covalent attachment of the expressed products as Fos-fusion proteins on the surface of filamentous phage particles, thus allowing the selective enrichment of phage-displaying IgG peptides in rounds of biopanning. The cDNAs encoding immunoreactive peptides can be recovered from phage particles by infection of bacterial cultures and then identified by DNA sequencing and database searches.

\section{Methods}

Patients. Sera from 55 vitiligo patients (22 male, 33 female; mean age: 48 years; age range: $14-77$ years) were 
used in this study. Of these patients, 41 had no other autoimmune disorder and no family history of autoimmune disease, and 14 had at least one other autoimmune disorder: autoimmune thyroid disease, 9 patients; alopecia areata, 3 patients; systemic lupus erythematosus (SLE), 1 patient; scleroderma, 1 patient. Sera from 28 healthy individuals ( 10 male, 18 female; age range: $21-59$ years; mean age: 34 years) were used as controls. As additional three sets of controls, sera from 20 patients ( 5 male, 15 female; mean age: 44 years; age range: $22-84$ years) with Graves disease (GD), 16 patients ( 7 male, 9 female; mean age: 48 years; age range: 26-77 years) with Addison disease (AD), and 15 patients with SLE (1 male, 14 female; mean age: 43 years; age range: $20-64$ years) were tested. All sera were kept frozen at $-20^{\circ} \mathrm{C}$. This study was approved by the Ethics Committee of the Northern General Hospital (Sheffield, United Kingdom), and all subjects gave informed consent.

Specific antisera. Rabbit polyclonal anti-melanin-concentrating hormone receptor 1 (anti-MCHR1) antiserum, MCHR11-S, was purchased from Alpha Diagnostic International Inc. (San Antonio, Texas, USA). Other rabbit polyclonal antisera against melanocytespecific proteins that were used as controls included antityrosinase ( $\alpha$ PEP7), antityrosinase-related protein-2 $(\alpha \mathrm{PEP} 8)$, and anti-Pmel17 (AZN-LAM).

Library construction. The pJuFo vector (18), a gift from R. Crameri (Swiss Institute of Allergy and Asthma Research, Davos, Switzerland), was modified to allow cloning of SfiI-restricted DNA fragments. Briefly, primers 5'CTAGAGGCCATTA TGGCCTGCAGGATCCGGCCGCCTCGGCCGGTAC3' and 5'CGGCCGAGGCGGCCG GATCCTGCAGGCCATAATGGCCT3' (Life Technologies Ltd., Paisley, United Kingdom) were treated with T4 polynucleotide kinase (Promega UK Ltd., Southampton, United Kingdom), annealed, and then ligated into $\mathrm{pJuFo}$ restricted with enzymes $\mathrm{XbaI}$ (Promega UK Ltd.) and KpnI (Promega UK Ltd.). Cultured skin melanocytes were a gift from S. MacNeil (Division of Clinical Sciences [North], University of Sheffield, Sheffield, United Kingdom). RNA was prepared from $2 \times 10^{6}$ cells using TRIZOL LS Reagent (Life Technologies Ltd.) as described in the manufacturer's instructions. Total RNA was then used in a SMART cDNA Library Construction Kit (CLONTECH Laboratories UK Ltd., Basingstoke, United Kingdom), according to the manufacturer's protocol, to prepare melanocyte cDNA fragments restricted with $S f i$ that were then ligated into the SfiI site of pJuFo. The melanocyte cDNA library was recovered by electroporation of Escherichia coli XL1-Blue cells (Stratagene, La Jolla, California, USA), as described by the manufacturer, and the library size estimated by plating out samples onto Luria Bertani (LB) agar (23) containing $50 \mu \mathrm{g} / \mathrm{ml}$ ampicillin.

To prepare a melanocyte cDNA phage-display library, the electroporated cells were incubated for 1 hour at $37^{\circ} \mathrm{C}$ before superinfection with $10^{12}$ plaque-forming units of VCMS13 helper phage (Stratagene) at room temperature for 30 minutes. The culture was subsequently transferred to $100 \mathrm{ml}$ of LB medium (23) supplemented with $50 \mu \mathrm{g} / \mathrm{ml}$ ampicillin, $10 \mu \mathrm{g} / \mathrm{ml}$ tetracycline, and $10 \mu \mathrm{g} / \mathrm{ml}$ kanamycin. After overnight incubation at $37^{\circ} \mathrm{C}$, the culture was centrifuged and phage precipitated from the supernatant as detailed previously (22). The phage was resuspended in $2-3 \mathrm{ml}$ of PBS and stored at $-20^{\circ} \mathrm{C}$. The phage titer was determined by infecting log-phase E. coli XL1-Blue with an aliquot of the phage-display library and then plating out samples onto LB agar containing $50 \mu \mathrm{g} / \mathrm{ml}$ ampicillin and $10 \mu \mathrm{g} / \mathrm{ml}$ tetracycline.

Isolation and biotinylation of IgG. IgG was isolated from the sera of patients by protein G-Sepharose 4 Fast Flow affinity column chromatography (Amersham Pharmacia Biotech Europe GmbH, Uppsala, Sweden). Eluted IgG fractions were concentrated using an Amicon Concentrator (Amicon Inc., Beverly, Massachusetts, USA). The concentrated IgG was filter sterilized with a Millex filter unit (Millipore Corp., Bedford, Massachusetts, USA), and the final concentration measured by photometry at $280 \mathrm{~nm}$. Biotinylation of the IgG was performed using EZ-Link Sulfo-NHS-LC-LC-Biotin (Pierce Chemical Co., Rockford, Illinois, USA), according to the manufacturer's protocol. All IgG samples were stored at $4^{\circ} \mathrm{C}$ until required.

Biopanning. A $15-\mu \mathrm{l}$ aliquot of biotinylated $\operatorname{IgG}$ was incubated with $200 \mu \mathrm{g}$ of Dynabeads M-280 streptavidin (Dynal Biotech ASA, Oslo, Norway), prepared according to the manufacturer in $235 \mu \mathrm{l}$ of sterile water, and incubated at $4^{\circ} \mathrm{C}$ for 30 minutes on a rotating platform to permit Ab-bead binding. The Ab sample used was a pool from ten vitiligo patients with each biotinylated $\operatorname{IgG}$ at a concentration of $2 \mathrm{mg} / \mathrm{ml}$. To block any nonspecific phage binding to the beads later in the procedure, $300 \mu \mathrm{l}$ of $2 \%$ dried milk in PBS containing $10 \%$ glycerol was added to the bead-IgG suspension, and incubation at $4^{\circ} \mathrm{C}$ continued for 1 hour. The bead-IgG complexes were separated from the blocking buffer using a Dynal Magnetic Particle Concentrator (Dynal Biotech ASA), washed twice, and finally resuspended in $150 \mu \mathrm{l}$ of PBS $/ 0.05 \%$ Tween 20 before the addition of a $100-\mu$ l sample of phage-display library containing $10^{10} \mathrm{CFU}$. The suspension was then incubated overnight at $4^{\circ} \mathrm{C}$ to allow interaction of the Abbead complexes with peptides displayed on the surface of the phage particles. The bead-IgG complexes were washed extensively with $\mathrm{PBS} / 0.05 \%$ Tween 20 to remove any unbound phage. Bound phage were eluted from the bead-IgG complexes with $150 \mu$ of $100 \mathrm{mM}$ $\mathrm{HCl}$ (adjusted to $\mathrm{pH} 2.2$ with solid glycine) and the beads magnetically separated from the supernatant that was neutralized by $9 \mu \mathrm{l}$ of $2 \mathrm{M}$ Tris- $\mathrm{HCl}$ ( $\mathrm{pH}$ 7.6). The phage suspension was subsequently used to infect $2 \mathrm{ml}$ of exponentially growing E. coli XL1-Blue (Stratagene) for 15 minutes at room temperature. Aliquots of the infected cells were then plated onto selective medium to allow the recovery of pJuFo phagemid. To gen- 
erate a phage-display library for a further round of selection, the infected E. coli XL1-Blue culture was superinfected with helper phage as described above. This first-round library enriched in phages displaying IgG-binding peptides was used in a second round of selective enrichment as detailed above. In all, three rounds of biopanning were undertaken.

Analysis of recombinant $P J u F o$ phagemid. Individual colonies, isolated from the third round of biopanning by plating out E. coli XL1-Blue infected with eluted phage, were grown and pJuFo phagemid prepared using the Wizard Minipreps DNA Purification System (Promega UK Ltd.). Phagemid DNA (50 ng samples) was subjected to 36 cycles of PCR amplification in a DNA Thermal Cycler (Perkin-Elmer Cetus, Norwalk, Connecticut, USA) with primers 1192 5'CCGCTGGATTGTTATTACTCGCTG $3^{\prime}$ and $15005^{\prime}$ TGCAAGGCGATT AAGTTGGGTAAC3' (Life Technologies Ltd.), which flank the $X b a \mathrm{I}-K p n I$ cloning sites in $\mathrm{pJuFo}$, using reaction conditions detailed previously (24). The PCR amplification products were analyzed by agarose gel electrophoresis (23) to confirm the presence of a cDNA insert and were purified according to a Wizard PCR Preps DNA Purification System (Promega UK Ltd.). Sequencing of PCR products was performed according to a Thermo Sequenase Cycle Sequencing Kit (USB Corp., Cleveland, Ohio, USA) with [ $\gamma^{32}$ P]ATP (ICN Pharmaceuticals Ltd., Basingstoke, United Kingdom) and primer 5'-CCGAAATCGCGAACCTGCTG-3' (Life Technologies Ltd.), which lies upstream of the $X b a I$ cloning site in $\mathrm{PJuFo}$. Sequenced DNA was subjected to electrophoresis in 6\% acrylamide/7 $\mathrm{M}$ urea gels with glycerol-tolerant gel buffer (USB Corp.). The cDNA sequences were compared with international databases using the BLAST network service of the National Center for Biotechnology Information (NCBI; Bethesda, Maryland, USA).

Cloning of buman MCHR1 CDNA. Total melanocyte RNA $(1 \mu \mathrm{g})$ was used to prepare cDNA in a $30-\mu \mathrm{l}$ reaction containing $10 \mathrm{mM}$ DTT, $1 \mathrm{mM}$ dNTPs (Promega UK Ltd.), $250 \mathrm{ng}$ random primers (Promega UK Ltd.), 600 U M-MLV RT (Promega UK Ltd.), and 1X M-MLV RT buffer (Promega UK Ltd.). The cDNA was subjected to PCR amplification using the conditions as described above with MCHR1-specific primers 5'TTGAATTCGCCGCCATGTTGTGTCCTTCCAAG3' and 5'AATCTAGACGCCTATCAGGTG CCTTTGCTTC3'. Restriction sites for $E c o$ RI and $X b a \mathrm{I}$ were incorporated into the forward and reverse primers, respectively, in order to allow subcloning of the 1,300-bp PCR amplification product into pcDNA3 (Invitrogen Ltd., Abingdon, United Kingdom) and subsequent expression of the MCHR1 cDNA from the $\mathrm{T} 7$ promoter in the vector. The recombinant plasmid, pcMCHR1, was purified with a QIAGEN Plasmid Maxi Kit (QIAGEN Ltd., Crawley, United Kingdom).

In vitro translation. In vitro translation of PcMCHR1 was performed according to the TnT T7 Coupled Reticulocyte Lysate System (Promega UK Ltd.) with translation-grade $\left[{ }^{35} \mathrm{~S}\right]$-methionine $(1,000 \mathrm{Ci} / \mathrm{mmol}$,
$10 \mathrm{mCi} / \mathrm{ml}$; Amersham Pharmacia Biotech Inc., Little Chalfont, United Kingdom). Glycosylation of MCHR1 was achieved using Canine Pancreatic Microsomal Membranes (Promega UK Ltd.) under conditions detailed by the manufacturer. SDS-PAGE of in vitro-translated MCHR1 was carried out in $12 \%$ polyacrylamide gels using standard protocols (23). Prestained SDS-PAGE standards (low range) were from Bio-Rad Laboratories Ltd. (Hemel Hempstead, United Kingdom). Gels were processed as detailed elsewhere (9) before autoradiography at $-70^{\circ} \mathrm{C}$.

Radiobinding assays. Assays were executed as detailed previously (9) with serum at a final dilution of 1:20 and with each sample in duplicate. Specific rabbit polyclonal antisera (MCHR11-S, $\alpha$ PEP7, $\alpha$ PEP8, AZN-LAM) were used at a final dilution of 1:100. An Ab index for each serum tested in the radiobinding assay was calculated as follows: counts per minute immunoprecipitated by tested serum divided by the mean counts per minute immunoprecipitated by 28 healthy control sera. Each serum was tested in at least two experiments, and the mean $\mathrm{Ab}$ index was calculated. The upper level of normal for the assay was calculated using the mean $\mathrm{Ab}$ index plus $3 \mathrm{SD}$ of the population of 28 healthy individuals. Any serum with an $\mathrm{Ab}$ index above the upper level of normal was designated as positive for $\mathrm{Ab}$ reactivity. The frequency of MCHR1 Ab's was compared between patient groups and controls using Fisher's exact test for $2 \times 2$ contingency tables. $P$ values less than 0.05 (two-tailed) were regarded as significant. For analysis of immunoprecipitated proteins by SDS-PAGE and autoradiography, the protein G-Sepharose Ab complexes were resuspended in $50 \mu$ l of SDS sample buffer (23), boiled, and the supernatant recovered for electrophoresis in a $12 \%$ polyacrylamide gel, which was processed as detailed above. For dilution experiments, vitiligo patient sera positive for MCHR1 Ab's were used in the radiobinding assay at final dilutions of 1:20, 1:50, $1: 100,1: 200,1: 500 ; 1: 1,000$, and 1:2,000.

Absorption experiments. The Chinese hamster ovary (CHO) cell line stably expressing MCHR1 has been reported previously (25). Cell membranes were prepared as described elsewhere (25). Vitiligo patient sera positive for MCHR1 Ab's and a pool of healthy con-

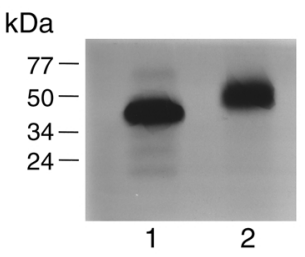

\section{Figure 1}

SDS-PAGE and autoradiography of in vitro-translated MCHR1. MCHR1 was produced in vitro in TnT T7 Coupled Reticulocyte Lysate System. To allow glycosylation of MCHR1, canine pancreatic microsomal membranes were added to the transcription-translation reaction. In vitro-translated MCHR1 (lane 1); In vitro-translated and -glycosylated MCHR1 (lane 2). 


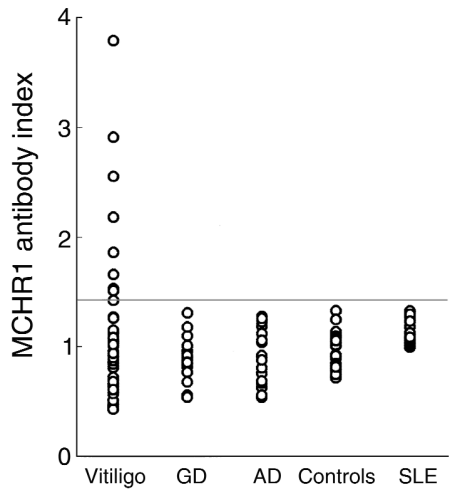

\section{Figure 2}

Radiobinding assays. Vitiligo sera $(n=55)$, GD patient sera $(n=20)$ AD patient sera $(n=16)$, healthy control sera $(n=28)$ and SLE patient sera $(n=15)$ were analyzed in radiobinding assays. The Ab index shown for each serum is the mean of at least two experiments. The gray line shows the upper level of normal for the radiobinding assay at an $\mathrm{Ab}$ index of 1.43 .

trols were incubated at $4^{\circ} \mathrm{C}$ for 12 hours with either $\mathrm{CHO}$ or CHO-MCHR1 cell membranes that were at a final concentration of $5 \mathrm{mg} / \mathrm{ml}$ of serum. After preincubation, the sera were used in the radiobinding assay with $\left[{ }^{35} \mathrm{~S}\right]$-labeled receptor as described above. Sera without prior absorption were assayed concurrently. $\mathrm{Ab}$ indices were calculated for each serum tested as follows: counts per minute immunoprecipitated by tested serum divided by the counts per minute immunoprecipitated by a pool of healthy control sera without preincubation with cell membranes. Samples were tested in two experiments, and the mean Ab index was calculated. Differences in $\mathrm{Ab}$ indices following preincubation with cell membranes were analyzed using the Student's $t$ test, and $P$ values less than 0.05 (two-tailed) were regarded as significant.

MCHR1-binding assays. MCHR1-binding assays were performed as described previously (25). Briefly, membranes $(10 \mu \mathrm{g})$ from $\mathrm{CHO}$ cells expressing MCHR1 were incubated with $100 \mu \mathrm{g}$ wheat germ agglutinin SPA beads (Amersham Life Sciences Inc., Arlington Heights, Illinois, USA) and 125 pM [ $\left.{ }^{125} \mathrm{I}\right]$-melaninconcentrating hormone (MCH) (NEN Life Science Products Inc., Boston, Massachusetts, USA) in a final volume of $200 \mu$ l of binding buffer $(25 \mathrm{mM}$ HEPES, 10 $\mathrm{mM} \mathrm{MgCl}_{2}, 10 \mathrm{mM} \mathrm{NaCl}, 5 \mathrm{mM} \mathrm{MnCl}_{2}, 0.1 \% \mathrm{BSA}$ ) for 2 hours at room temperature. Nonspecific binding was determined by addition of $1 \mathrm{mM}$ unlabeled $\mathrm{MCH}$ in the binding incubation. Bound $\left[{ }^{125} \mathrm{I}\right]-\mathrm{MCH}$ was detected using a TOPCOUNT microplate scintillation counter (Packard Instrument Co., Downers Grove, Illinois, USA). Inhibition of [ $\left.{ }^{125} \mathrm{I}\right]-\mathrm{MCH}$ binding was analyzed by preincubation of cell membranes with IgG, prepared from either healthy individuals or MCHR1 Ab-positive vitiligo patients at a final concentration of $2 \mathrm{mg} / \mathrm{ml}$. A percentage inhibition of $\mathrm{MCH}$ binding was calculated for each IgG sample tested as 100: (cpm bound after incubation of CHO-MCHR1 membranes with IgG sample/cpm bound without incubation of CHO-MCHR1 membranes $\times 100)$. Differences in the percentage of inhibition of $\mathrm{MCH}$ binding following incubation of CHO-MCHR1 cell membranes with vitiligo and control IgG samples were analyzed using the Student's $t$ test. $P$ values less than 0.05 (two-tailed) were regarded as significant.

\section{Results}

Construction of the melanocyte cDNA phage-display library. The primary size of the pJuFo melanocyte cDNA library was $10^{6}$ independent clones. Following amplification with helper phage, a stock phage-display library with a titer of $10^{11} \mathrm{CFU} / \mathrm{ml}$ was produced. To determine the frequency of recombinant phagemid in the phage-display library, $40 \mathrm{CFU}$ were grown and the pJuFo phagemid isolated from each culture. Analysis of the phagemids by PCR amplification with primers 1192 and 1500 indicated that the phage-display library contained recombinant pJuFo phagemids at an approximate frequency of $90 \%$. The cloned fragments ranged in size from 800 to 2,500 bp in length. This phage-display library was used for the selective enrichment of IgG-binding proteins from vitiligo patients.

Enrichment of phage displaying IgG-binding peptides. The melanocyte cDNA phage-display library was subjected to three rounds of biopanning against a pool of biotinylated IgG from ten vitiligo patients who had no other autoimmune disease. After the third round of enrichment, $30 \mathrm{CFU}$ were grown. Phagemid DNA was isolated from each culture and analyzed by PCR amplification with 1192 and 1500 primers. The PCR amplification products were purified and sequenced to aid identification of the cDNAs by BLAST searches of international databases. MCHR1 was encoded by one of the cDNAs recovered from the biopanning process. The MCHR1 cDNA was subsequently cloned into pcDNA3.

In vitro translation and immunoreactivity of MCHR1. Plasmid pcMCHR1 was translated in vitro. The quality of the in vitro-translated radiolabeled receptor was evaluated by SDS-PAGE and autoradiography. This revealed a protein product with an estimated molecu-

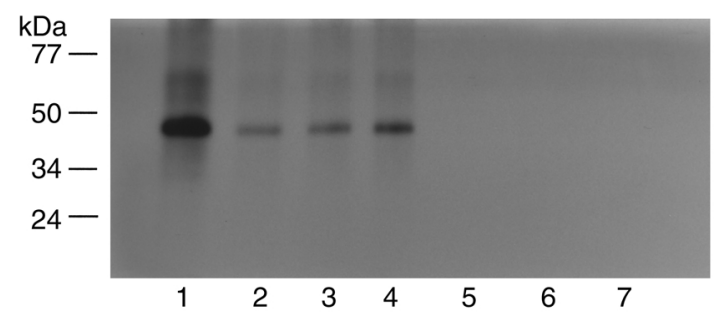

\section{Figure 3}

SDS-PAGE and autoradiography of MCHR1 immunoprecipitated with either MCHR1 Ab-positive vitiligo or healthy control sera. MCHR1 was produced in vitro in TnT T7 Coupled Reticulocyte Lysate System and then used in radiobinding assays with either vitiligo or healthy control sera. In vitro-translated MCHR1 (lane 1); MCHR1 immunoprecipitated with MCHR1 Ab-positive vitiligo sera (lanes 2-4); MCHR1 immunoprecipitated with healthy control sera (lanes 5-7). 


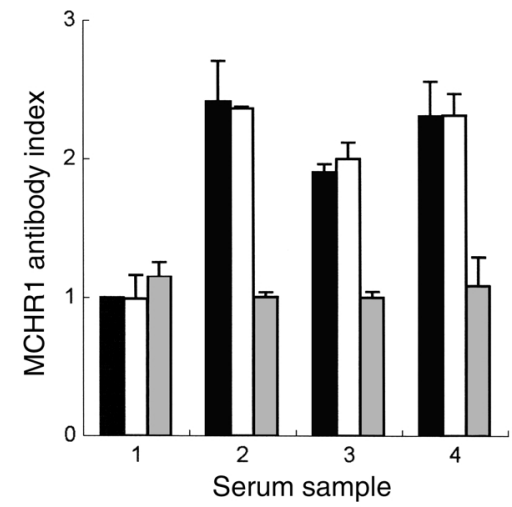

Figure 4

Absorption experiments. MCHR1 Ab-positive sera $(n=3)$ and a pool of healthy control sera $(n=6)$ were preabsorbed with either $\mathrm{CHO}$ or $\mathrm{CHO}-\mathrm{MCHR} 1$ cell membranes before analysis in the radiobinding assay. The results of two experiments are shown as the mean $A b$ index and SD. A pool of control sera (sample 1) and MCHR1 Abpositive vitiligo patient sera (samples 2-4), each without preincubation (black bars), with preincubation with $\mathrm{CHO}$ cell membranes (white bars) and with preincubation with $\mathrm{CHO}-\mathrm{MCHR} 1$ cell membranes (gray bars).

lar weight of $45 \mathrm{kDa}$ (Figure 1), a size that is in close agreement with the molecular weight of $39 \mathrm{kDa}$ predicted from the amino acid sequence of the protein (26). On the addition of canine pancreatic microsomal membranes to the in vitro translation reaction, a protein band with an increased molecular weight of 50 $\mathrm{kDa}$ was visible (Figure 1 ). We assume this to be glycosylated MCHR1. The immunoreactivity of the in vitro-translated radiolabeled MCHR1 was analyzed in radiobinding assays using rabbit polyclonal antisera. The mean Ab indices and SD for MCHR11-S, $\alpha$ PEP7, $\alpha \mathrm{PEP} 8$, and AZN-LAM were $9.16 \pm 4.01,0.97 \pm 0.05$, $1.33 \pm 0.42$, and $0.86 \pm 0.34$, respectively, thereby demonstrating the immunoreactivity of labeled MCHR1 to the receptor-specific antiserum.

Radiobinding assays. Sera from 55 vitiligo patients, 28 healthy controls, 15 SLE patients, $16 \mathrm{AD}$ patients, and 20 GD patients were tested for immunoreactivity to MCHR1 in radiobinding assays. For each serum, an Ab index was assigned, this being the mean $\mathrm{Ab}$ index of at least two experiments (Figure 2). The mean $\mathrm{Ab}$ indices plus or minus SD of the control, GD, AD, SLE, and vitiligo groups were: $0.95 \pm 0.16,0.88 \pm 0.19,0.92 \pm 0.26$, $1.13 \pm 0.11$, and $1.07 \pm 0.62$, respectively. The upper level of normal for the radiobinding assay (mean $\mathrm{Ab}$ index plus $3 \mathrm{SD}$ of 28 healthy controls) was estimated as an $\mathrm{Ab}$ index of 1.43 (Figure 2). Any serum with an $\mathrm{Ab}$ index above this level was considered positive for MCHR1 Ab's. On this basis, none of the healthy individuals was positive for MCHR1 Ab's. Sera from 20 patients with GD, 16 with $\mathrm{AD}$, and 15 with SLE were all negative for $\mathrm{Ab}$ 's to the receptor. Of the vitiligo patient sera examined, 9 of 55 (16.4\%) were considered positive for MCHR1 Ab's, and the frequency of receptor Ab's in the vitiligo patient group was significantly greater than normal $(P=0.025)$. The use of glycosylated MCHR1 in the radiobinding assay did not alter the $\mathrm{Ab}$ index of any of the vitiligo or healthy control sera tested. SDS-PAGE was used to check that the radioactivity immunoprecipitated by each of the MCHR1 Ab-positive sera in the radiobinding assays was due to $\left[{ }^{35} \mathrm{~S}\right]$-labeled receptor. Figure 3 indicates that the Ab-positive sera immunoprecipitated a protein band of the correct size when compared with in vitro-translated MCHR1. In dilution experiments, three MCHR1 Ab-positive vitiligo sera showed saturated binding up to a dilution of 1:50 (data not shown).

Absorption experiments. Absorption experiments were carried out to assess the specificity of Ab binding to MCHR1 in Ab-positive vitiligo sera $(n=3)$. A pool of sera obtained from healthy subjects $(n=6)$ was used as a control. Following preabsorption with $\mathrm{CHO}-$ MCHR1 cell membranes, the $\mathrm{Ab}$ indices (Figure 4) for the vitiligo sera were significantly different when compared with the $\mathrm{Ab}$ indices for the vitiligo sera without preabsorption $(P=0.002)$ and the vitiligo sera with preincubation with $\mathrm{CHO}$ cell membranes $(P=0.001)$. In contrast, $\mathrm{Ab}$ binding to the receptor was not affected by preincubation of the vitiligo serum samples with $\mathrm{CHO}$ cell membranes: there was no significant difference between the $\mathrm{Ab}$ indices (Figure 4 ) measured with and without preincubation $(P=0.949)$. The results suggest that $A b$ reactivity in the vitiligo patient sera was specifically absorbed by expressed MCHR 1 in the CHO cell membranes.

MCHR1-binding assays. The effect of IgG from MCHR1 Ab-positive vitiligo patients $(n=6)$ and healthy controls $(n=6)$ upon the binding of $\mathrm{MCH}$ to the receptor was analyzed in MCHR1-binding assays. The mean percentage of inhibition plus or minus SD of MCH binding for the vitiligo and control $\operatorname{IgG}$ groups was $45 \% \pm 4 \%$ and $16 \% \pm 14 \%$, respectively (Figure 5). The IgGs from MCHR1 Ab-positive vitiligo patients significantly

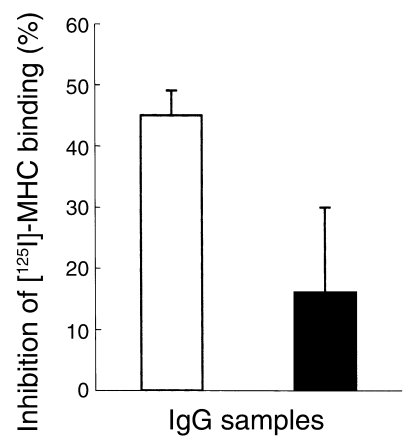

\section{Figure 5}

MCHR1-binding assays. CHO-MCHR1 cell membranes were preincubated with IgG from either vitiligo patients $(n=6)$ or controls $(n=6)$ before binding of $\left[{ }^{125} \mathrm{I}\right]-\mathrm{MCH}$ was measured in MCHR1-binding assays. The mean percentage of inhibition and SD of [ $\left.{ }^{125} \mathrm{l}\right]-\mathrm{MCH}$ binding is shown for MCHR1 Ab-positive vitiligo IgG (white bar) and control IgG samples (black bar). The IgGs from MCHR1 Ab-positive vitiligo patients significantly inhibited $\mathrm{MCH}$ binding when compared with the IgG samples from control subjects $(P=0.001)$. 
Table 1

Comparison of MCHR1 Ab-positive and Ab-negative vitiligo patients

\begin{tabular}{|c|c|c|}
\hline & \multicolumn{2}{|c|}{ Vitiligo patients $(n=55)$} \\
\hline & $\begin{array}{l}\text { MCHR1 Ab-positive } \\
\quad(n=9)\end{array}$ & $\begin{array}{l}\text { MCHR1 Ab-negative } \\
\quad(n=46)\end{array}$ \\
\hline $\begin{array}{l}\text { Mean age at onset of disease } \\
\text { (age range) }\end{array}$ & 38 years $(<1-73$ years $)$ & 35 years (6-70 years) \\
\hline $\begin{array}{l}\text { Mean age at serum sample } \\
\text { (age range) }\end{array}$ & 47 years ( $23-75$ years) & 48 years $(14-77$ years \\
\hline Mean disease duration (range) & 10 years ( $1-37$ years) & 13 years $(<1-49$ years \\
\hline \multicolumn{3}{|l|}{ Sex: } \\
\hline $\begin{array}{l}\text { Male }{ }^{\mathrm{A}} \\
\text { Female }^{\mathrm{A}}\end{array}$ & $\begin{array}{l}5 / 9(56 \%) \\
4 / 9(44 \%)\end{array}$ & $\begin{array}{l}17 / 46(37 \%) \\
29 / 46(63 \%)\end{array}$ \\
\hline Other autoimmune disorder ${ }^{B}$ & $2 / 9(22 \%)^{B}$ & $12 / 46(26 \%)$ \\
\hline \multicolumn{3}{|l|}{ Vitiligo subtype: } \\
\hline Periorificial $^{A}$ & $1 / 9(11 \%)$ & $1 / 46(2 \%)$ \\
\hline Symmetrical $^{\mathrm{A}}$ & $6 / 9(67 \%)$ & $36 / 46(78 \%)$ \\
\hline Symmetrical and periorificial ${ }^{A}$ & $1 / 9(11 \%)$ & $4 / 46(9 \%)$ \\
\hline Symmetrical and segmental ${ }^{\mathrm{A}}$ & $1 / 9(11 \%)$ & $2 / 46(4 \%)$ \\
\hline Segmental ${ }^{A}$ & $0 / 9(0 \%)$ & $1 / 46(2 \%)$ \\
\hline Focal ${ }^{A}$ & $0 / 9(0 \%)$ & $1 / 46(2 \%)$ \\
\hline Universal ${ }^{A}$ & $0 / 9(0 \%)$ & $1 / 46(2 \%)$ \\
\hline
\end{tabular}

AThe characteristics of the MCHR1 Ab-positive and Ab-negative vitiligo patients were compared using Fisher's exact test. All $P$ values were more than 0.05 , indicating no significant difference between the two groups with respect to the characteristics analyzed. ${ }^{B}$ Autoimmune thyroid disease was diagnosed in both patients.

inhibited $\mathrm{MCH}$ binding when compared with the IgG samples from control subjects $(P=0.001)$.

Analysis of MCHR1 Ab-positive vitiligo patients. The details of the MCHR1 Ab-positive and Ab-negative vitiligo patients are compared in Table 1 . No association was evident between the presence of MCHR1 Ab's and either the age of the patient at time of serum sampling, the age of the patient at onset of disease, the duration of the disease, or the gender of the patients. In addition, the clinical subtype of vitiligo did not appear to be related to the occurrence of receptor Ab's, and three patients who had active vitiligo were all negative for Ab's against MCHR1. Furthermore, the presence of an autoimmune disorder did not correlate with MCHR1 Ab reactivity. Among the MCHR1 Ab-positive vitiligo patients, only one in nine was positive for $\mathrm{Ab}$ 's to tyrosinase, TRP-1, TRP-2, and Pmel17, and one had $\mathrm{Ab}$ reactivity to Pmel17 alone. None was positive for Ab's to SOX10 (Table 2).

Longitudinal data with regard to the persistence of MCHR1 Ab reactivity was available for only four of the MCHR1 Ab-positive vitiligo patients. For one patient, MCHR1 Ab's could still be detected in a serum sample taken 12 months after the sample that was initially tested. For the remaining three patients, receptor Ab's could be demonstrated in sera taken at 5, 6, and 8 months, respectively, after the sample that was analyzed at the outset of the study.

\section{Discussion}

Employing phage-display technology with a melanocyte cDNA phage-display library, we have identified MCHR1 as a novel target of autoantibody reactivity in vitiligo patients. MCHR1 cDNA was recovered following the third round of biopanning against the melanocyte cDNA phage-display library with a pool of vitiligo IgG from ten patients. Radiobinding assays using $\left[{ }^{35} \mathrm{~S}\right]-$ labeled MCHR1 were subsequently used to confirm immunoreactivity against the receptor in sera from patients with vitiligo. Of the 55 vitiligo sera analyzed, Ab's to the receptor were detected in $16.4 \%$, whereas AD, GD, SLE, and control sera showed no reactivity, indicating a high disease-associated specificity. Glycosylation of the receptor did not alter the $\mathrm{Ab}$ binding index of any of the vitiligo patient sera tested, suggesting that any MCHR1 epitope recognized by the Ab-positive sera was not altered by glycosylating the protein. Furthermore, this finding indicated that vitiligo patient sera negative for MCHR1 Ab's did not recognize either glycosylated epitopes or epitopes that might be altered by posttranslational processing of the receptor. Similar findings have been documented for the thyroid autoantigens thyroglobulin and thyroid peroxidase, because deglycosylation of these molecules had no effect on autoantibody binding (27). It is possible that some receptor Ab's were not detected in the radiobinding assay employed here using recombinant MCHR1. Indeed, the most prevalent Ab's to the thyroid-stimulating hormone receptor (TSHR) in Graves disease patients only react with native TSHR as they recognize epitopes that depend on the structure of the protein (28). The incidence of MCHR1 Ab's may be increased if native receptor was used to analyze vitiligo sera for Ab's that recognize conformational epitopes.

Analysis of the MCHR1 Ab-positive vitiligo patients revealed no obvious association between the presence of receptor Ab's and either patient age at the time of serum sampling, patient age at the onset of disease, sex, disease duration, or vitiligo subtype. Furthermore, the occurrence of MCHR1 Ab's did not appear to correlate with the presence of an autoimmune disorder. In contrast, our previous studies indicated that Ab's to the melanocyte-specific enzymes TRP-1, TRP-2, and

\section{Table 2}

Incidence of antimelanocyte Ab's in MCHR1 Ab-positive patients

\begin{tabular}{|c|c|c|c|c|c|c|c|c|c|}
\hline & \multicolumn{9}{|c|}{ MCHR1 Ab-positive vitiligo patient } \\
\hline & 1 & 2 & 3 & 4 & 5 & 6 & 7 & 8 & 9 \\
\hline \multicolumn{10}{|c|}{ cidence of Ab's to: } \\
\hline Tyrosinase & - & - & - & - & - & - & - & - & + \\
\hline TRP-1 & - & - & - & - & - & - & - & - & + \\
\hline TRP-2 & - & - & - & - & - & - & - & - & + \\
\hline Pmel17 & - & - & - & - & - & - & - & + & + \\
\hline SOX10 & - & ND & - & - & - & - & - & - & ND \\
\hline
\end{tabular}

Ab reactivity was analyzed in radiobinding assays as previously detailed for tyrosinase (9), TRP-1 (10), TRP-2 (11), Pmel17 (13), and SOX10 (14). -, negative for $A b$ reactivity; + , positive for $A b$ reactivity; $N D$, not determined. 
Pmel17 were detected only in vitiligo patients who had an autoimmune disorder $(10,11,13)$, and $\mathrm{Ab}$ reactivity to tyrosinase and SOX10 was identified predominantly in patients with autoimmune disease $(9,14)$. In addition, the presence of MCHR1 Ab's did not appear to be related to the occurrence of humoral responses to tyrosinase, TRP-1, TRP-2, and Pmel17. Only two of the MCHR1 Ab-positive vitiligo sera exhibited reactivity to these melanocyte antigens. Two other vitiligo patients tested in this study were positive for Ab's to all the aforementioned melanogenic autoantigens, and one patient was positive for Ab's to tyrosinase. However, none of them had Ab's to MCHR1.

MCHR1 is a G-coupled receptor for the neuropeptide $\mathrm{MCH}$ that is involved in the regulation of food intake and energy balance $(29,30)$. The receptor is stimulated by $\mathrm{MCH}$ to mobilize intracellular $\mathrm{Ca}^{2+}$ and reduce forskolin-elevated cAMP levels $(29,30)$. In a diverse range of physiological roles, $\mathrm{MCH}$ can act as a functional antagonist of $\alpha$-melanocyte-stimulating hormone $(\alpha-\mathrm{MSH})$ that binds to the melanocortin- 1 receptor $(29,30)$ and, initially, $\mathrm{MCH}$ was described in teleost fish where the activity of the hormone decreased skin pigmentation (31). Recently, MCHR1 expression has been reported in human melanocytes and melanoma cell lines (32). Further study revealed that $\mathrm{MCH}$ both reduced the increase in cAMP that occurs in response to $\alpha-\mathrm{MSH}$ and partly inhibited the induction of melanogenesis by $\alpha-\mathrm{MSH}$ in human melanocytes (32). This suggested that the $\mathrm{MCH} / \mathrm{MCHR} 1$ signaling pathway might function in the regulation of melanocytes and hence melanin production. Because MCHR1 is exposed on the cell surface, it could be accessible to autoantibodies that might adversely affect the functioning of the receptor, leading to the disruption of normal melanocyte behavior. With regard to this possibility, experiments undertaken in this study demonstrated that IgG from MCHR1 Ab-positive vitiligo patients has an inhibitory affect upon the binding of $\mathrm{MCH}$ to its receptor. Other autoimmune disorders that result directly from autoantibody production against surface receptors include Graves disease, hypothyroidism, and myasthenia gravis. In autoimmune thyroid disease, the G protein-coupled TSHR, present on the surface of thyroid follicular cells, is the target of autoantibodies that can either stimulate TSHR, leading to an increase in the synthesis of thyroid hormones and the subsequent clinical symptoms of hyperthyroidism, or inhibit the binding of TSH, leading to hypothyroidism (33).

Exactly how anti-MCHR1 Ab's arise remains to be determined. A genetic predisposition to autoimmunity or cross-reacting antigens, expressed on either other cells or on infecting microorganisms, might elicit their production. Alternatively, MCHR1 Ab's might result from an immune response following damage to pigment cells induced by other mechanisms. Because the expression of MCHR1 is not limited to melanocytes and the receptor is found within the central nervous system $(29,30)$ and in keratinocytes $(34)$, the selective destruc- tion of pigment cells, as observed in vitiligo, might result from the relative sensitivity of melanocytes to immune-mediated injury as compared with, for example, fibroblasts and keratinocytes (35).

In summary, the use of phage-display technology has enabled the isolation of a novel autoantigen in vitiligo, MCHR1. Although Ab's in vitiligo patients are apparently most commonly directed against melanocyte antigens on the surface of the cell $(5,6)$, this is the first study, to our knowledge, that has specifically identified a surface receptor as an autoantigen in vitiligo. Previously, only intracellular melanocyte proteins such as tyrosinase have been reported as autoantigens in this depigmenting skin disorder (7-14). Furthermore, in vitiligo patients, Ab's to MCHR1 appear to be more frequent than those to the melanogenic autoantigens tyrosinase, TRP-1, TRP-2, and Pmel17, at least in our experience (9-11, 13). Finally, Ab's against MCHR1 might indicate the presence of autoreactive anti-MCHR1 T lymphocytes that are capable of destroying pigment cells, a possibility that requires further investigation.

\section{Acknowledgments}

This research was supported by grants from the Vitiligo Society, the British Skin Foundation (276), and the Special Trustees for the Former United Sheffield Hospitals' Charitable Funds (87794) to E.H. Kemp, D.J. Gawkrodger, and A.P. Weetman.

1. Ochi, Y., and DeGroot, L.J. 1969. Vitiligo in Graves' disease. Ann. Intern. Med. 71:935-940.

2. Macaron, C., et al. 1977. Vitiligo and juvenile diabetes mellitus. Arch. Dermatol. 113:1515-1517.

3. Naughton, G.K., Eisinger, M., and Bystryn, J.-C. 1983. Antibodies to normal human melanocytes in vitiligo. J. Exp. Med. 158:246-251.

4. Ogg, G.S., Dunbar, P.R., Romero, P., Chen, J.-L., and Cerundolo, V. 1998. High frequency of skin-homing melanocyte-specific cytotoxic T lymphocytes in autoimmune vitiligo. J. Exp. Med. 188:1203-1208.

5. Cui, J., Harning, R., Henn, M., and Bystryn, J.-C. 1992. Identification of pigment cell antigens defined by vitiligo antibodies. J. Invest. Dermatol. 98:162-165.

6. Cui, J., Arita, Y., and Bystryn, J.-C. 1995. Characterisation of vitiligo antigens. Pigment Cell Res. 8:53-59.

7. Baharav, E., et al. 1996. Tyrosinase as an autoantigen in patients with vitiligo. Clin. Exp. Immunol. 105:84-88.

8. Song, Y.H., et al. 1994. The role of tyrosinase in autoimmune vitiligo. Lancet. 344:1049-1052.

9. Kemp, E.H., Gawkrodger, D.J., MacNeil, S., Watson, P.F., and Weetman, A.P. 1997. Detection of tyrosinase autoantibodies in vitiligo patients using ${ }^{35} \mathrm{~S}$-labelled recombinant human tyrosinase in a radioimmunoassay. J. Invest. Dermatol. 109:69-73.

10. Kemp, E.H., Waterman, E.A., Gawkrodger, D.J., Watson, P.F., and Weetman, A.P. 1998. Autoantibodies to tyrosinase-related protein-1 detected in the sera of vitiligo patients using a quantitative radiobinding assay. Br. J. Dermatol. 139:798-805.

11. Kemp, E.H., Gawkrodger, D.J., Watson, P.F., and Weetman, A.P. 1997. Immunoprecipitation of melanogenic enzyme autoantigens with vitiligo sera: evidence for cross-reactive autoantibodies to tyrosinase and tyrosinase-related protein-2 (TRP-2). Clin. Exp. Immunol. 109:495-500.

12. Okamoto, T., et al. 1998. Anti-tyrosinase-related protein-2 immune response in vitiligo and melanoma patients receiving active-specific immunotherapy. J. Invest. Dermatol. 111:1034-1039.

13. Kemp, E.H., Gawkrodger, D.J., Watson, P.F., and Weetman, A.P. 1998. Autoantibodies to human melanocyte-specific protein Pmel17 in the sera of vitiligo patients: a sensitive and quantitative radioimmunoassay (RIA). Clin. Exp. Immunol. 114:333-338.

14. Hedstrand, H., et al. 2001. The transcription factors SOX9 and SOX10 are vitiligo autoantigens in autoimmune polyendocrine syndrome type I. J. Biol. Chem. 276:35390-35395. 
15. Van den Wijngaard, R., et al. 2000. Local immune response in skin of generalised vitiligo patients. Lab. Invest. 80:1299-1309.

16. Lang, K.S., et al. 2001. HLA-A2 restricted, melanocyte-specific CD8+ T lymphocytes detected in vitiligo patients are related to disease activity and are predominantly directed against MelanA/MART1. J. Invest. Dermatol. 116:891-897.

17. Palermo, B., et al. 2001. Specific cytotoxic T lymphocyte responses against MelanA/MART1, tyrosinase and Gp100 in vitiligo by the use of major histocompatibility complex/peptide tetramers: the role of cellular immunity in the etiopathogenesis of vitiligo. J. Invest. Dermatol. 117:326-332.

18. Crameri, R., and Suter, M. 1993. Display of biologically active proteins on the surface of filamentous phages: a cDNA cloning system for selection of functional gene products linked to the genetic information responsible for their production. Gene. 137:69-75.

19. Crameri, R., Jaussi, R., Menz, G., and Blaser, K. 1994. Display of expression products of cDNA libraries on phage surfaces. Eur. J. Biochem. 226:53-58.

20. Crameri, R., et al. 1996. Humoral and cell-mediated autoimmunity in allergy to Aspergillus fumigatus. J. Exp. Med. 184:265-270.

21. Crameri, R., and Blaser, K. 1996. Cloning Aspergillus fumigatus allergens by the pJuFo filamentous phage-display system. Int. Arch. Allergy Immunol. 110:41-45.

22. Lindborg, M., et al. 1999. Selective cloning of allergens from the skin colonising yeast Malassezia furfur by phage surface display technology. $J$. Invest. Dermatol. 113:156-161.

23. Sambrook, J., Fritsch, E.F., and Maniatis, T. 1989. Molecular cloning: a laboratory manual. 2nd edition. Cold Spring Harbor Laboratory Press. New York, New York, USA.

24. Kemp, E.H., Waterman, E.A., Gawkrodger, D.J., Watson, P.F., and Weetman, A.P. 2001. Molecular mapping of epitopes on melanocyte-specific protein Pmel17 which are recognised by autoantibodies in patients with vitiligo. Clin. Exp. Immunol. 124:509-515.
25. Hawes, B.E., et al. 2000. The melanin-concentrating hormone receptor couples to multiple $G$ proteins to activate diverse intracellular signalling pathways. Endocrinology. 141:4524-4532.

26. Kolakowski, L.F., et al. 1996. Characterisation of a human gene related to genes encoding somatostatin receptors. FEBS Lett. 398:253-258.

27. Kiso, Y., Furmaniak, C., Morteo, C., and Smith, B.R. 1992. Analysis of carbohydrate residues on human thyroid peroxidase (TPO) and thyroglobulin $(\mathrm{Tg})$ and effects of deglycosylation, reduction and unfolding on autoantibody binding. Autoimmunity. 12:259-269.

28. Morgenthaler, N.G., et al. 1999. Direct binding of thyrotropin receptor autoantibody to in vitro translated thyrotropin receptor: a comparison to radioreceptor assay and thyroid stimulating bioassay. Thyroid. 9:467-475.

29. Chambers, J., et al. 1999. Melanin-concentrating hormone is the cognate ligand for the orphan G-protein-coupled receptor SLC-1. Nature. 400:261-265.

30. Saito, Y., et al. 1999. Molecular characterisation of the melanin-concentrating-hormone receptor. Nature. 400:265-269.

31. Kawauchi, H., Kawazoe, I., Tsubokawa, M., Kishida, M., and Baker, B.I. 1983. Characterisation of melanin-concentrating hormone in chum salmon pituitaries. Nature. 305:321-323.

32. Hoogdijn, M.J., Ancans, J., and Thody, A.J. 2001. Melanin-concentrating hormone may act as a paracrine inhibitor of melanogenesis. Br.J. Dermatol. 144:651-677.

33. Rapoport, B., and McLachlan, S.M. 2001. Thyroid autoimmunity. J. Clin Invest. 108:1253-1259. DOI:10.1172/JCI200114321.

34. Burgaud, J.-L., Pootsi, R., Fehrentz, J.-A., Martinez, J., and Nahon, J.-L. 1997. Melanin-concentrating hormone binding sites in human SVK14 keratinocytes. Biochem. Biophys. Res. Commun. 241:622-629.

35. Norris, D.A., et al. 1988. Enhanced susceptibility of melanocytes to different immunologic effector mechanisms in vitro: potential mechanisms for post-inflammatory hypopigmentation and vitiligo. Pigment Cell Res. 1(Suppl.):113-123. 